\title{
Predictors of chronicity and the discriminative value of the new ACR/EULAR rheumatoid arthritis classification criteria in an untreated polyarthritis cohort with less than 6 weeks of disease duration
}

\author{
A F Mourão $0^{1 *+}$, H Canhão ${ }^{1,2 \dagger}$, R A Moura ${ }^{1}$, R Cascão ${ }^{1}$, P Weinmann ${ }^{1}$, A Rodrigues ${ }^{1,2}$, J Pereira ${ }^{1,2}$, C Resende ${ }^{2}$, \\ S Capela ${ }^{2}$, J A Pereira da Silva², J E Fonseca ${ }^{1,2}$
}

From 5th European Workshop on Immune-Mediated Inflammatory Diseases

Sitges-Barcelona, Spain. 1-3 December 2010

\section{Aims}

1. To find predictors of progression to RA in a cohort of patients with a very recent onset polyarthritis. 2 . To test the performance of the new ACR/EULAR criteria for the classification of RA in this population.

\section{Patients}

Patients with polyarthritis with less than 6 weeks of duration were enrolled during a period of 4 years. They were not exposed previously to corticosteroids or DMARDs. All patients were prospectively followed-up in order to establish a definitive clinical diagnosis. A protocol was applied, including demographic and clinical data, and a blood sample was collected to assess ESR, RF and ACPA levels, before any treatment was started.

\section{Results}

Thirty-seven patients were included. During the follow up most of the patients evolved into RA (57\%). The median age of the patients of the RA-group was similar to the median age of the non-RA group (median (IQR) 47 (31-58.5) vs 43 (34-69) years, $\mathrm{p}=0.74)$. At the initial visit the DAS 28 in the RA group was significantly higher than in the non-RA group, as well as the VAS and the number of swollen joints. The ESR values did not differ significantly between RA and non-RA groups (Table 1).

\footnotetext{
† Contributed equally

'Rheumatology Research Unit, Instituto de Medicina Molecular, Lisbon, Portugal

Full list of author information is available at the end of the article
}

Table 1 Baseline differences between patients who latter evolved into RA (RA-group), comparing to who did not (non-RA group)

\begin{tabular}{llll}
\hline Parameter & RA & Non-RA & p-value \\
\hline DAS28 & $6.2(4.9-7.3)$ & $4.8(3.8-5.9)$ & $0.016^{*}$ \\
VAS & $60(50-89.5)$ & $46(30-60)$ & $0.045^{*}$ \\
Swollen joints & $8(3-20)$ & $2(0-9.7)$ & $0.042^{*}$ \\
Tender joints & $10(3-20)$ & $5.5(2-17.7)$ & 0.202 \\
Total involved joints & $12(8-18)$ & $10.5(3.5-18)$ & 0.380 \\
CRP & $2.74(1.7-4.3)$ & $2.1(0.1-4.8)$ & 0.810 \\
ESR & $37(26.5-63.5)$ & $25(19.5-60)$ & 0.419 \\
HAQ & $1.9(1-2)$ & $0.87(0.25-1)$ & $0.020^{*}$ \\
\hline
\end{tabular}

${ }^{*} p$-value <0.05; DAS28: Disease Activity Score 28; VAS: Visual Analogue Scale; ESR: Erythrocyte sedimentation rate; HAQ: Health Assessment Questionnaire; RA: Rheumatoid arthritis. Values are expressed as median and interquartile range: median (IQR).

Among the 21 RA patients, 21 were evaluated for RF and 17 for ACPA in the first visit. Of these, 45\% presented RF and 37.5\% presented ACPA. RF and ACPA were not detectable in any of the patients who did not evolve to RA.

According to the new criteria for the classification of RA, the mean total score of the RA group at baseline was significantly higher than the non-RA group (median (IQR) 6 (4.5-8) vs 4.5 (2.2-6), $p=0,007)$. Interestingly, the highest score reached in the non-RA patients was 6 points, the cut-off point to classify patients as having RA.

\section{Conclusion}

In our cohort a high DAS28 score, a high number of swollen joints, a low functional status and the presence 
of RF or ACPA are associated with the future evolution into RA. The new ACR/EULAR criteria for the classification of RA seem to identify the majority of patients that will evolve to RA.

\section{Author details}

${ }^{1}$ Rheumatology Research Unit, Instituto de Medicina Molecular, Lisbon, Portugal. ${ }^{2}$ Rheumatology Dept., Santa Maria Hospital, Lisbon, Portugal.

Published: 25 November 2010

doi:10.1186/1479-5876-8-S1-P65

Cite this article as: Mourão et al:: Predictors of chronicity and the discriminative value of the new ACR/EULAR rheumatoid arthritis classification criteria in an untreated polyarthritis cohort with less than 6 weeks of disease duration. Journal of Translational Medicine 2010 8(Suppl 1):P65

Submit your next manuscript to BioMed Central and take full advantage of:

- Convenient online submission

- Thorough peer review

- No space constraints or color figure charges

- Immediate publication on acceptance

- Inclusion in PubMed, CAS, Scopus and Google Scholar

- Research which is freely available for redistribution

Submit your manuscript at www.biomedcentral.com/submit 